\title{
Planar Capacitated Dominating Set is $W[1]$-hard
}

\author{
Hans L. Bodlaender ${ }^{1}$, Daniel Lokshtanov ${ }^{2}$, and Eelko Penninkx ${ }^{1}$ \\ 1 Department of Information and Computing Sciences, Universiteit Utrecht, PO Box 80.089, 3508TB \\ Utrecht, The Netherlands. Emails: \{hansb, penninkx\}@cs.uu.nl \\ 2 Department of Informatics, University of Bergen, PO Box 7803, N-5020 Bergen, Norway. \\ Email: daniello@ii.uib.no
}

\begin{abstract}
Given a graph $G$ together with a capacity function $c: V(G) \rightarrow \mathbb{N}$, we call $S \subseteq V(G)$ a capacitated dominating set if there exists a mapping $f:(V(G) \backslash S) \rightarrow S$ which maps every vertex in $(V(G) \backslash S)$ to one of its neighbors such that the total number of vertices mapped by $f$ to any vertex $v \in S$ does not exceed $c(v)$. In the Planar CAPACitated Dominating Set problem we are given a planar graph $G$, a capacity function $c$ and a positive integer $k$ and asked whether $G$ has a capacitated dominating set of size at most $k$. In this paper we show that Planar Capacitated Dominating Set is $W[1]$-hard, resolving an open problem of Dom et al. [IWPEC, 2008]. This is the first bidimensional problem to be shown $W[1]$-hard. Thus Planar Capacitated Dominating Set can become a useful starting point for reductions showing parameterized intractablility of planar graph problems.
\end{abstract}

\section{Introduction}

In the Dominating Set problem we are given a graph $G$ and asked for the smallest set of vertices such that every vertex in the graph either belongs to this set or has a neighbor which does. This basic problem in algorithms and complexity has been studied extensively, and finds applications in various domains. Dominating Set has a special place in parameterized complexity $[5,8,12]$. It is the most well-known $W[2]$-complete problem and is a standard starting point for reductions that show intractability of parameterized problems [5]. Even though the Dominating SET problem is a fundamentally hard problem in the parameterized $W$-hierarchy, it has been used as a benchmark problem for developing sub-exponential time FPT algorithms [1,3,10], and also for obtaining linear kernels on planar graphs $[2,8,11,12]$, and more generally, graphs that exclude a fixed graph $H$ as a minor.

Different applications of Dominating SET have initiated studies of different generalizations and variations of the problem. These include Connected Dominating Set, Partial Dominating Set, and Capacitated Dominating Set to name a few. In this paper we focus on one such generalization, namely CAPACITATED DominatING SET. Given a graph $G$ together with a capacity function $c: V(G) \rightarrow \mathbb{N}$, we call $S \subseteq V(G)$ a capacitated dominating set if there exists a mapping $f:(V(G) \backslash S) \rightarrow S$ which maps every vertex in $(V(G) \backslash S)$ to one of its neighbors such that the total number of vertices mapped by $f$ to any vertex $v \in S$ does not exceed $c(v)$. The CAPACitated Dominating Set problem is defined as follows.

Capacitated Dominating Set (CDS): Given a graph $G$, a capacity function $c$ and a positive integer $k$, determine whether there exists a capacitated dominating set $S$ of $G$ containing at most $k$ vertices. 
Dom et al. initiated the study of CDS from the perspective of Parameterized Complexity, and showed that CDS is $W[1]$-hard parameterized by solution size and the treewidth of the input graph [4]. Like Dominating SET, CDS has become a useful source for showing $W$-hardness, especially when the parameter is the structure of the input graph $[7,9]$. It has been recently used to show the first $W$-hardness results for problems parameterized by the cliquewidth of the input graph [9].

Many graph problems that are $W$-hard in general turn out to be FPT when restricted to planar graphs. This is true for Dominating SET and many of its variants, and hence it is very natural to consider the parameterized complexity of PlanaR CAPACITATED Dominating SET, the restriction of CDS to planar graphs. For most planar graph problems, an FPT algorithm can be obtained by combining a combinatorial bound on the treewidth of non-trivial instances with a dynamic programming algorithm for graphs of bounded treewidth. In fact for most problems restricted to planar graphs we have subexponential time parameterized algorithms using bidimensionality theory [3]. PCDS, however, is an exception to this rule. In particular, it can easily be shown by using bidimensionality that any planar graph that has a capacitated dominating set of size at most $k$ has treewidth $O(\sqrt{k})$. On the other hand, Dom et al. showed that CDS is $W[1]$-hard when parameterized by solution size and the treewidth of the input graph [4]. Thus, bidimensionality alone was not enough to tackle this problem and it was an intriguing question whether PCDS could still turn out to be FPT by a non-trivial use of planarity. We show that these hopes were futile by giving a $W$ [1]-hardness reduction for PCDS. Planar Capacitated Dominating Set is the first bidimensional problem to be shown $W[1]$-hard. We believe that Planar Capacitated Dominating Set can become a useful starting point for reductions showing parameterized intractablility of planar graph problems.

\section{Preliminaries}

We will work with both undirected and directed graphs. Given a graph $G$, the vertex set of $G$ is $V(G)$ and the edge set of $G$ is $E(G)$. For a graph $G, n=|V(G)|$ and $m=|E(G)|$. With $N_{G}(u)$ we denote all vertices that are adjacent to $u$ and the degree of $u$ is $d_{G}(u)=\left|N_{G}(u)\right|$. Let $f$ be the function associated with a capacitated dominating set $S$. Given $u \in S$ and $v \in V \backslash S$, we say that $u$ dominates $v$ if $f(v)=u$; moreover, every vertex $u \in S$ dominates itself. Note that the capacity of a vertex $v$ only limits the number of neighbors that $v$ can dominate, that is, a vertex $v \in S$ can dominate $c(v)$ of its neighbors plus $v$ itself.

For a directed graph $D$ the node set of $D$ is $N(D)$ and the arc set of $D$ is $A(D)$. For a node $u, N_{D}^{+}(u)=\{v: u v \in A\}$ is the set of outneighbours of $u, N_{D}^{-}(u)=\{v: v u \in A\}$ is the set of inneighbours of $u$ and $N_{D}(u)=N_{D}^{+}(u) \cup N_{D}^{-}(u)$ is the set of neighbours of $u$. We define $d_{D}^{+}(u)=\left|N_{D}^{+}(u)\right|, d_{D}^{-}(u)=\left|N_{D}^{-}(u)\right|$ and $d_{D}(u)=\left|N_{D}(u)\right|$ to be the outdegree, indegree and degree of $u$ respectively.

We use the notions of a parameterized problem, Fixed Parameter Tractability, hardness for the complexity class $W[1]$ and our hardness proofs involve F PT-reductions. For an introduction to these notions, the reader is referred to the textbooks $[5,8,12]$. For ease of reference we provide the definition of FPT-reductions here. 
Definition 1. $[5,8,12]$ Let $A, B$ be parameterized problems. We say that $A$ is FPTreducible to $B$ if there is an algorithm $\Phi$ which transforms $(x, k)$ into $\left(x^{\prime}, g(k)\right)$ in time $f(k) \cdot|x|^{\alpha}$, where $f, g: \mathbb{N} \rightarrow \mathbb{N}$ are arbitrary functions and $\alpha$ is a constant independent of $|x|$ and $k$, so that $(x, k) \in A$ if and only if $\left(x^{\prime}, g(k)\right) \in B$.

It is well known that if $A$ is hard for $W[1]$ and $A$ is FPT-reducible to $B$, then $B$ is also $W[1]$-hard $[5,8,12]$.

\section{PCDS is W[1]-hard parameterized by solution size}

In this section we show that PCDS is $W[1]$-hard when parameterized by solution size. We reduce from Multi-Color Clique, a restriction of the $k$-Clique problem.

Multi-Color Clique (MCC) Given an integer $k$ and a connected undirected graph $G=(V[1] \cup V[2] \cdots \cup V[k], E)$ such that for every $i$ the vertices of $V[i]$ induce an independent set, is there a $k$-clique $C$ in $G$ ?

For $i \leq k$ the sets $V[i]$ are called color classes of $G$. Since each color class forms an independent set, a $k$-clique in $G$ must contain exactly one vertex from each color class. For two distinct integers $i, j$ between 1 and $k$ the set $E[i, j]$ is the set of edges of $G$ with one endpoint in $V[i]$ and the other in $V[j]$. The Multi-Color Clique problem is known to be W[1]-hard [6] and is used as a starting point for many hardness reductions.

We will reduce to a slightly modified version of Planar CaPaCitated Dominating Set, Planar Marked Capacitated Dominating Set (PMCDS) where we mark some vertices and demand that all marked vertices must be in the dominating set. We can then reduce from PMCDS to Planar Capacitated Dominating Set by attaching $k+1$ leaves to each marked vertex and increasing the capacity of each marked vertex by $k+1$. It is easy to see that the new instance has a $k$-capacitated dominating set if and only if the original one had a $k$-capacitated dominating set that contained all marked vertices, and that this operation preserves planarity of the graph. Thus, to prove that Planar Capacitated Dominating Set is $W$ [1]-hard when parameterized by solution size, it is sufficient to prove that PMCDS is. We will show how given an instance $(G, k)$ of Multicolor Clique, we can build an instance $\left(H, c, k^{*}\right)$ of PMCDS such that $k^{*}=O\left(k^{3}\right)$ and $G$ has a clique of size $k$ if and only if $H$ has a capacitated dominating set of size $k^{*}$.

The reduction to PMCDS goes via an intermediate problem as well. We name this problem the Planar ARC Supply problem, (PAS). In PAS we are given a planar digraph $D=(N, A)$ with $|N(D)|+|A(D)|=k$, no loops and no double arcs. Every node $u \in N$ has a demand $\zeta(u)$ and every arc $u v \in A$ has a list $L(u v)$ of ordered integer pairs, called the supply pairs of $u v$. The task is to decide whether there is a function $f_{a}: A \rightarrow \mathbb{N}$ and a function $f_{b}: A \rightarrow \mathbb{N}$ such that for every arc $u v \in A$ we have $\left(f_{a}(u v), f_{b}(u v)\right) \in L(u v)$ and for every node $u \in N$ we have that $\zeta(u) \leq$ $\sum_{v \in N^{+}(u)} f_{a}(u v)+\sum_{v \in N^{-}(u)} f_{b}(v u)$. In essense we are asked to pick a supply pair from the list of every arc such that for every vertex the arcs incident to it are able to cover the demand of the vertex. Therefore the pair of fuctions $f_{a}$ and $f_{b}$ are called a supply selection and a supply selection is called satisfying if the demand of all vertices is met. 


\subsection{Identification Numbers.}

Given an instance $(G=(V[1] \cup \ldots \cup V[k], E), k)$ to MCC every vertex and every edge of $G$ gets two pairs of identification numbers. Every vertex gets one pair of small and one pair of medium identification numbers and every edge gets one pair of small and one pair of large identification numbers. For the vertices these identification numbers are defined as follows.

- Every vertex $v$ gets assigned a unique number $I_{S}^{U}(v)$ between 1 and $n$ as its small up-ID.

- The small down-ID of $v$ is $I D_{S}^{D}(v)=n^{2}-I D_{S}^{U}(v)$.

- The medium up-ID of $v$ is $I D_{M}^{U}(v)=n^{3} \cdot I D_{S}^{U}(v)$.

- The medium down-ID of $v$ is $I D_{M}^{D}(v)=n^{3} \cdot I D_{S}^{D}(v)$.

For edges the identification numbers are defined similarly. In particular:

- Every edge $u v$ of $G$ gets assigned a unique number $I D_{S}^{U}(u v)$ between 1 and $m$ as its small up-ID.

- The small down-ID of $u v$ is $I D_{S}^{D}(u v)=n^{2}-I D_{S}^{U}(u v)$.

- The large up-ID of $u v$ is $I D_{L}^{U}(u v)=n^{6} \cdot I D_{S}^{U}(u v)$.

- The large down-ID of $u v$ is $I D_{L}^{D}(u v)=n^{6} \cdot I D_{S}^{D}(u v)$.

Observe that $2 m<n^{2}$ and that therefore all small down-ID's are larger than all small up-ID's. We also define the huge numbers $\mathcal{U}=n^{20}-n^{19}, \mathcal{D}=n^{20}+n^{19}$ and $\mathcal{M}=n^{20}$. The intuition behind using different "sizes" of identification numbers is that one can think of the PAS instance we cosntruct as a set of wires in which signals are travelling, with the identification numbers being the amplitude of the signal sent. Signals that are of completely different orders of magnitude do not interfer with one another, in the sense that when looking at "large" signals the signals of smaller order seem like unimportant noise. However when the "large" signal is fixed, the "meduim" signals become important etc. We will use the identification numbers first to give a reduction from MCC to PAS. We will then reduce the obtained PAS instance to an instance of PMCDS. Some of the identification numbers are vital for the first reduction, some for the second, and some for both.

\subsection{Reduction to Planar Arc Supply.}

Given an instance $(G=(V[1] \cup \ldots \cup V[k], E), k)$ to $\mathrm{MCC}$ we now construct an instance of Planar Arc Supply. For every color class $i$ between 1 and $k$ we make a horizontal cycle $C_{H}^{i}$. This is a directed cycle of length $12\left(\begin{array}{c}k \\ 2\end{array}\right)$, oriented clockwise. For every pair of color classes $i, j$ between 1 and $k$ such that $i<j$ we make a vertical cycle $C_{V}^{i, j}$. This is a directed cycle of length $12 k$, oriented clockwise. We arrange the cycles in a grid, with the vertical cycles corresponding to vertical lines on the grid and the horizontal cycles corresponding to the horizontal lines on the grid. Every horizontal cycle intersects with every vertical cycle in exactly four points. Following a cycle, there are exactly three edges from one intersection point to the next. This concludes the construction of the directed graph $D$. Observe that the graph $D$ itself depends only on $k$. For a construction of $D$ for $k=3$ see Figure 1 . 


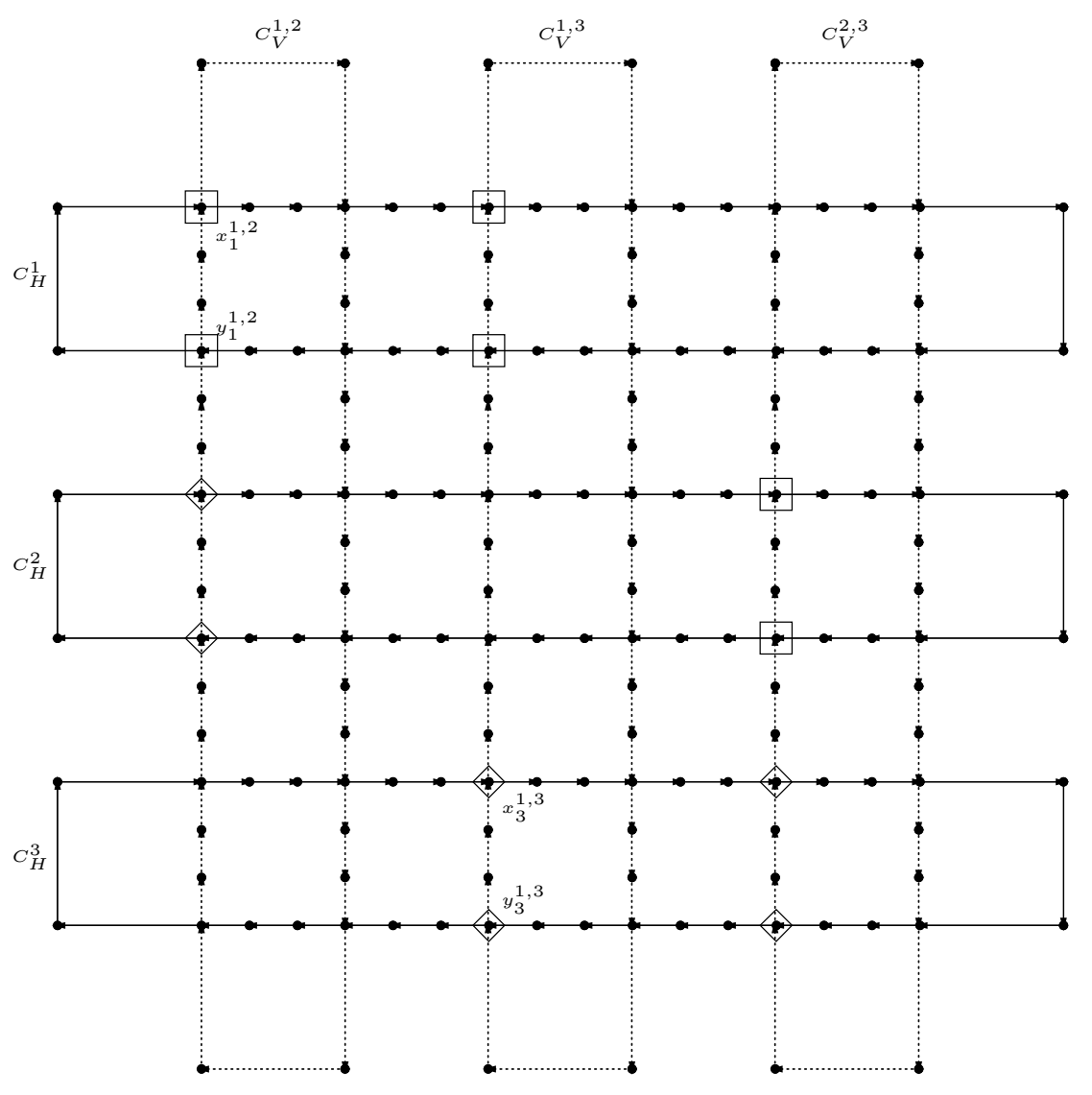

Fig. 1. The construction of $D$ for $k=3$. Horizontal cycles are shown in full lines, vertical in dotted lines. The left-matching vertices are surrounded by a square, the right-matching vertices are surrounded by a diamond.

The number of arcs in $D$ is $k \cdot 12\left(\begin{array}{l}k \\ 2\end{array}\right)+\left(\begin{array}{l}k \\ 2\end{array}\right) \cdot 12 k$. The number of nodes in $D$ is $k \cdot 12\left(\begin{array}{l}k \\ 2\end{array}\right)+\left(\begin{array}{l}k \\ 2\end{array}\right) \cdot 12 k-4 k \cdot\left(\begin{array}{l}k \\ 2\end{array}\right)$. Hence $k^{\prime}=|N(D)|+|A(D)|=44 k\left(\begin{array}{l}k \\ 2\end{array}\right)=O\left(k^{3}\right)$.

The horizontal cycle $C_{H}^{t}$ and the vertical cycle $C_{V}^{i, j}$ intersect in exactly four nodes. The top-left intersection node is called $x_{t}^{i, j}$ and the bottom left intersection node is called $y_{t}^{i, j}$. The nodes $x_{t}^{i, j}$ and $y_{t}^{i, j}$ such that $i=t$ are called left-matching nodes and the nodes $x_{t}^{i, j}$ and $y_{t}^{i, j}$ such that $j=t$ are called right-matching nodes. An arc in a horizontal cycle is called a horizontal arc and an arc in a vertical cycle is called a vertical arc. An arc whose endpoint is a left-matching node is also called left-matching and similarly an arc whose endpoint is a right-matching node is called left-matching. If an arc or node is left-matching or right-matching then it is also a matching arc (node).

We now describe the demands of all the nodes and the supply lists of all the arcs of $D$. Observe that every node $v$ of $G$ has degree either 2 or 4 . The demand of every vertex of degree 2 in a horizontal cycle is $2 M+n^{5}$. The demand of every vertex of degree 2 in a vertical cycle is $2 M+n^{8}$ and the demand of every degree 4 vertex is $4 M+n^{8}+n^{5}$. The description of the supply-lists conclude the description of the PAS instance. Every 
pair in a supply list of a horizontal arc will correspond to a vertex of $G$ while every pair in a supply list of a vertical arc will correspond to an edge of $G$.

The intuition is that each horizontal cycle $C_{H}^{i}$ encodes the choice of which vertex in $V[i]$ will be in the clique. Each vertical cycle $C_{V}^{i, j}$ encodes the choice of which edge of $E[i, j]$ will be in the clique. The large identification numbers are used in all arcs of the vertical cycles to encode this choice of edges. The medium identification numbers are used in all arcs of the horizontal cycles to encode the choice of vertices. In the intersection of the horizontal cycle $C_{H}^{i}$ and the vertical cycle $C_{V}^{i, j}$ we use the left-matching vertices $x_{i}^{i, j}$ and $y_{i}^{i, j}$ to make sure that the vertex selected in the horizontal cycle $C_{H}^{i}$ and the edge selected in the vertical cycle $C_{V}^{i, j}$ are incident. Similarly, in the intersection of the horizontal cycle $C_{H}^{j}$ and the vertical cycle $C_{V}^{i, j}$ we use the right-matching vertices $x_{j}^{i, j}$ and $y_{j}^{i, j}$ to make sure that the vertex selected in the horizontal cycle $C_{H}^{j}$ and the edge selected in the vertical cycle $C_{V}^{i, j}$ are incident. The incidence check is performed using small identification numbers. The huge numbers $U$ and $D$ are always present in all supply pairs. These numbers are used in second stage of the reduction (from PAS to PMCDS) and do not play a role in the first part. We now formally describe the supply-lists.

- For every non-matching horizontal arc $u v$ of the cycle $C_{H}^{i}$, for every $a \in V[i]$ there is a pair $\left(\mathcal{U}+I D_{M}^{U}(a), \mathcal{D}+I D_{M}^{D}(a)\right)$ in $L(u v)$

- For every matching horizontal arc $u v$ pointing at a node $x_{i}^{i, j}$, for every $a \in V[i]$ there is a pair $\left(\mathcal{U}+I D_{M}^{U}(a), \mathcal{D}+I D_{M}^{D}(a)+I D_{S}^{D}(a)\right)$ in $L(u v)$.

- For every matching horizontal arc $u v$ pointing at a node $y_{i}^{i, j}$, for every $a \in V[i]$ there is a pair $\left(\mathcal{U}+I D_{M}^{U}(a), \mathcal{D}+I D_{M}^{D}(a)-I D_{S}^{D}(a)\right)$ in $L(u v)$

- For every non-matching vertical arc $u v$ of the cycle $C_{V}^{i, j}$, for every $a b \in E[i, j]$ there is a pair $\left(\mathcal{U}+I D_{L}^{U}(a b), \mathcal{D}+I D_{L}^{D}(a b)\right)$ in $L(u v)$.

- For every left-matching vertical arc $u v$ pointing at a node $x_{i}^{i, j}$, for every $a \in V[i]$ and $b \in V[j]$ such that $a b \in E$ there is a pair $\left(\mathcal{U}+I D_{L}^{U}(a b), \mathcal{D}+I D_{L}^{D}(a b)-I D_{S}^{D}(a)\right)$ in $L(u v)$.

- For every left-matching vertical arc $u v$ pointing at a node $y_{i}^{i, j}$, for every $a \in V[i]$ and $b \in V[j]$ such that $a b \in E$ there is a pair $\left(\mathcal{U}+I D_{L}^{U}(a b), \mathcal{D}+I D_{L}^{D}(a b)+I D_{S}^{D}(a)\right)$ in $L(u v)$.

- For every right-matching vertical arc $u v$ pointing at a node $x_{i}^{i, j}$, for every $a \in V[i]$ and $b \in V[j]$ such that $a b \in E$ there is a pair $\left(\mathcal{U}+I D_{L}^{U}(a b), \mathcal{D}+I D_{L}^{D}(a b)-I D_{S}^{D}(b)\right)$ in $L(u v)$.

- For every right-matching vertical arc $u v$ pointing at a node $y_{i}^{i, j}$, for every $a \in V[i]$ and $b \in V[j]$ such that $a b \in E$ there is a pair $\left(\mathcal{U}+I D_{L}^{U}(a b), \mathcal{D}+I D_{L}^{D}(a b)+I D_{S}^{D}(b)\right)$ in $L(u v)$.

Lemma 1. If $G$ contains a clique $C$ of size $k$ then $D$ has a satisfying supply selection.

Proof. Any $k$-clique in $G$ must contain exactly one vertex from each set $V[i]$ and exactly one edge from each set $E[i, j]$. Let $c_{i}$ be the vertex in $C \cap C_{i}$. For every $i$ between 1 and $k$ and every horizontal arc in $C_{H}^{i}$ select the supply pair corresponding to $c_{i}$. For every pair $i, j$ such that $i<j$ and every vertical arc in $C_{V}^{i, j}$ select the supply pair corresponding to the edge $c_{i} c_{j}$. The case analysis below shows that the demand of all vertices is met. 
- For every vertex with degree 2 on the horizontal cycle $C_{H}^{i}$, demand is $2 M+n^{5}$, and total supply is $D+I D_{M}^{D}\left(c_{i}\right)+\mathcal{U}+I D_{M}^{U}\left(c_{i}\right)=2 \mathcal{M}+n^{5}$.

- For every vertex with degree 2 on the vertical cycle $C_{H}^{i, j}$, demand is $2 M+n^{8}$, and total supply is $D+I D_{L}^{D}\left(c_{i} c_{j}\right)+\mathcal{U}+I D_{L}^{U}\left(c_{i} c_{j}\right)=2 \mathcal{M}+n^{8}$.

- For every non-matching vertex with degree 4 lying on the horizontal cycle $C_{H}^{t}$ and vertical cycle $C_{V}^{i, j}$, demand is $4 M+n^{8}+n^{5}$, and total supply is $D+I D_{M}^{D}\left(c_{t}\right)+\mathcal{U}+$ $I D_{M}^{U}\left(c_{t}\right)+\mathcal{D}+I D_{L}^{D}\left(c_{i} c_{j}\right)+\mathcal{U}+I D_{L}^{U}\left(c_{i} c_{j}\right)=4 \mathcal{M}+n^{8}+n^{5}$.

- For every left-matching vertex $x_{i}^{i, j}$, demand is $4 M+n^{8}+n^{5}$, and total supply is $D+I D_{M}^{D}\left(c_{i}\right)+I D_{S}^{D}\left(c_{i}\right)+\mathcal{U}+I D_{M}^{U}\left(c_{i}\right)+\mathcal{D}+I D_{L}^{D}\left(c_{i} c_{j}\right)-I D_{S}^{D}\left(c_{i}\right)+\mathcal{U}+I D_{L}^{U}\left(c_{i} c_{j}\right)=$ $4 \mathcal{M}+n^{8}+n^{5}$.

- For every left-matching vertex $y_{i}^{i, j}$, demand is $4 M+n^{8}+n^{5}$, and total supply is $D+I D_{M}^{D}\left(c_{i}\right)-I D_{S}^{D}\left(c_{i}\right)+\mathcal{U}+I D_{M}^{U}\left(c_{i}\right)+\mathcal{D}+I D_{L}^{D}\left(c_{i} c_{j}\right)+I D_{S}^{D}\left(c_{i}\right)+\mathcal{U}+I D_{L}^{U}\left(c_{i} c_{j}\right)=$ $4 \mathcal{M}+n^{8}+n^{5}$.

- For every right-matching vertex $x_{j}^{i, j}$, demand is $4 M+n^{8}+n^{5}$, and total supply is $D+I D_{M}^{D}\left(c_{j}\right)+I D_{S}^{D}\left(c_{j}\right)+\mathcal{U}+I D_{M}^{U}\left(c_{j}\right)+\mathcal{D}+I D_{L}^{D}\left(c_{i} c_{j}\right)-I D_{S}^{D}\left(c_{j}\right)+\mathcal{U}+I D_{L}^{U}\left(c_{i} c_{j}\right)=$ $4 \mathcal{M}+n^{8}+n^{5}$.

- For every right-matching vertex $y_{j}^{i, j}$, demand is $4 M+n^{8}+n^{5}$, and total supply is $D+I D_{M}^{D}\left(c_{j}\right)-I D_{S}^{D}\left(c_{j}\right)+\mathcal{U}+I D_{M}^{U}\left(c_{j}\right)+\mathcal{D}+I D_{L}^{D}\left(c_{i} c_{j}\right)+I D_{S}^{D}\left(c_{j}\right)+\mathcal{U}+I D_{L}^{U}\left(c_{i} c_{j}\right)=$ $4 \mathcal{M}+n^{8}+n^{5}$.

Lemma 2. If $D$ has a satisfying supply selection then $G$ contains a clique $C$ of size $k$.

Proof. Every pair in a supply list of a horizontal arc corresponds to a vertex of $G$ while every pair in a supply list of a vertical arc corresponds to an edge of $G$. Hence the satisfying supply selection of $D$ represents a choice of an edge of $G$ for every arc in a vertical cycle, and a choice of a vertex for every arc in a horizontal cycle. Consider two consecutive $\operatorname{arcs} u v$ and $v w$ on a vertical cycle $C_{V}^{i, j}$ and let $a$ be the edge of $G$ selected at $u v$ and $b$ be the edge selected at $v w$. We prove that $I D_{L}^{U}(b) \geq I D_{L}^{U}(a)$. Suppose for contradiction that $I D_{L}^{U}(b)<I D_{L}^{U}(a)$. If $v$ has degree 2 then demand is $2 M+n^{8}$ and supply is $D+I D_{L}^{D}(a)+\mathcal{U}+I D_{L}^{U}(b)<2 \mathcal{M}+n^{8}$. Observe that since $I D_{L}^{U}(a)=n^{6} \cdot I D_{S}^{U}(a)$ we have that if $I D_{L}^{U}(b)<I D_{L}^{U}(a)$ then $I D_{L}^{U}(b)+n^{6} \leq I D_{L}^{U}(a)$. Thus, if $v$ has degree 4 then $v$ 's demand is $2 M+n^{8}+n^{5}$ and the total supply at $v$ is at $\operatorname{most} D+I D_{L}^{D}(a)+n^{2}+\mathcal{U}+I D_{L}^{U}(b)+\mathcal{U}+n^{5}+\mathcal{D}+n^{5} \leq 4 \mathcal{M}+n^{8}+2 n^{5}+n^{2}-n^{6}<$ $4 \mathcal{M}+n^{8}+n^{5}$. Hence $I D_{L}^{U}(b) \geq I D_{L}^{U}(a)$. Since this holds for every pair of consecutive arcs on the vertical cycle $C_{V}^{i, j}$, all arcs on the cycle $C_{V}^{i, j}$ select the same edge of $G$.

We now prove that a similar observation holds for the horizontal cycles, that is, that all arcs on the horizontal cycle $C_{H}^{i}$ select the same vertex of $G$. Consider two consecutive arcs $u v$ and $v w$ on a horizontal cycle $C_{H}^{i}$ and let $a$ be the vertex of $G$ selected at $u v$ and $b$ be the vertex selected at $v w$. We prove that $I D_{M}^{U}(b) \geq I D_{M}^{U}(a)$. Suppose for contradiction that $I D_{M}^{U}(b)<I D_{M}^{U}(a)$. If $v$ has degree 2 then $v$ 's demand is $2 M+n^{5}$ and the supply at $v$ is $U+I D_{M}^{D}(a)+\mathcal{D}+I D_{M}^{U}(b)<2 \mathcal{M}+n^{5}$. Now, suppose $v$ has degree 4 , then the demand of $v$ is $4 M+n^{8}+n^{5}$. Observe that if $I D_{M}^{U}(b)<I D_{M}^{U}(a)$ then $I D_{M}^{U}(b)+n^{3} \leq I D_{M}^{U}(a)$. Also, since all arcs on the vertical cycle containing $v$ select the same edge of $G$, the vertical arcs incident to $v$ supply $v$ with at most $2 M+n^{8}+n^{2}$. Hence the total supply at $v$ is at most $2 M+n^{8}+n^{2}+\mathcal{D}+I D_{M}^{D}(a)+n^{2}+\mathcal{U}+I D_{M}^{U}(b) \leq$ 
$4 \mathcal{M}+n^{8}+n^{5}+2 n^{2}-n^{3}<4 \mathcal{M}+n^{8}+n^{5}$. Hence $I D_{L}^{U}(b) \geq I D_{L}^{U}(a)$. Since this holds for every pair of consecutive arcs on the horizontal cycle $C_{H}^{i}$, all arcs on the cycle $C_{H}^{i}$ select the same vertex of $G$.

Thus every horizontal cycle $C_{H}^{i}$ selects a vertex $c_{i} \in V[i]$ and every vertical cycle $C_{V}^{i, j}$ selects an edge $e_{i, j} \in E[i, j]$. It remains to prove that for every $i, j, e_{i, j}$ is incident to both $c_{i}$ and to $c_{j}$. We prove that $e_{i, j}$ is incident to $c_{i}$. In particular, let $c_{i}^{\prime}$ be the vertex in $V[i]$ incident to $e_{i, j}$ in $V[i]$. We prove that $I D_{S}^{D}\left(c_{i}\right)=I D_{S}^{D}\left(c_{i}^{\prime}\right)$. Suppose that $I D_{S}^{D}\left(c_{i}\right)<I D_{S}^{D}\left(c_{i}^{\prime}\right)$. Then the supply at $x_{i}^{i, j}$ is at most $D+I D_{M}^{D}\left(c_{i}\right)+I D_{S}^{D}\left(c_{i}\right)+\mathcal{U}+$ $I D_{M}^{U}\left(c_{i}\right)+\mathcal{D}+I D_{L}^{D}\left(e_{i, j}\right)-I D_{S}^{D}\left(c_{i}^{\prime}\right)+\mathcal{U}+I D_{L}^{U}\left(e_{i, j}\right)<4 \mathcal{M}+n^{8}+n^{5}$, a contradiction. Similarly if $I D_{S}^{D}\left(c_{i}\right)>I D_{S}^{D}\left(c_{i}^{\prime}\right)$ then the supply at $y_{i}^{i, j}$ is at most $D+I D_{M}^{D}\left(c_{i}\right)-$ $I D_{S}^{D}\left(c_{i}\right)+\mathcal{U}+I D_{M}^{U}\left(c_{i}\right)+\mathcal{D}+I D_{L}^{D}\left(e_{i, j}\right)+I D_{S}^{D}\left(c_{i}^{\prime}\right)+\mathcal{U}+I D_{L}^{U}\left(e_{i, j}\right)<4 \mathcal{M}+n^{8}+n^{5}$. Hence $I D_{S}^{D}\left(c_{i}\right)=I D_{S}^{D}\left(c_{i}^{\prime}\right)$ and $e_{i, j}$ is incident to $c_{i}$. The proof that $e_{i, j}$ is incident to $c_{j}$ is similar. This proves that $\left\{c_{1}, \ldots, c_{k}\right\}$ is a clique in $G$.

\subsection{Reduction to Planar Marked Capacitated Dominating Set}

We now show how to transform an instance $D, k^{\prime}$ of Planar ArC Supply constructed from a Multi-Color Clique instance $G, k$ as in Section to an instance $H, k^{*}$ of Planar Marked Capacitated Dominating Set. To build $H$ we start with the node set $N(D)$ and make every vertex of $N(D)$ marked. For every arc $u v$ of $D$ we make a gadget between $u$ and $v$ in $H$. In particular, for an arc $u v \in A(D)$, for every pair of integers $(p, q) \in L(u, v)$ we add a vertex $w$ to $H$, make $w$ adjacent to $u$, add $p$ vertices of degree 2 adjacent to $u$ and $w$ and add $q$ vertices of degree 2 adjacent to $w$ and $v$. We call the vertex $w$ is a list vertex. This concludes the construction of the graph $H$. Since $D$ is planar and the gadget we add to $H$ for every arc of $D$ is planar, $H$ is planar as well. Every marked vertex $v$ of $H$ is also a vertex in $D$. The capacity of $v$ in $H$ is set to $d_{H}(v)-\zeta(v)-d_{D}^{+}(v)$, that is, the degree of $v$ in $H$, minus $v$ 's demand in $D$ and minus $v$ 's outdegree in $D$. For all unmarked vertices, their capacity in $H$ is equal to their degree in $H$. Finally, $k^{*}=|N(D)|+|A(D)|$. This concludes the construction of the PlanaR Marked Capacitated Dominating Set instance $\left(H, k^{*}\right)$.

Lemma 3. If D has a satisfying supply selection then $H$ has a capacitated dominating set of size $k^{*}$

Proof. We build a capacitated dominating set $S$ of $H$. First we insert all the marked vertices of $H$ in $S$. For every arc $u v$ of $D$ we add a list vertex $w$ to $S$, namely the list vertex that corresponds to the supply pair in $L(u v)$ that was selected by the satisfying supply selection of $D$. The size of $S$ is $|N(D)|+|A(D)|=k^{*}$. We now prove that $S$ is a capacitated dominating set of $H$.

First, observe that the marked vertices of $H$ form a dominating set of $H$, so $S$ is a dominating set of $H$. Now, every unmarked vertex in $S$ has capacity equal to its degree, so all unmarked vertices in $S$ dominate all their neighbours. We now prove that for every marked vertex $u$, the number of yet undominated neighbours of $u$ is at most the capacity of $u$. The number of neighbours of $u$ that already have been dominated is at least $\zeta(u)$. The number of neighbours of $u$ that are in $S$ is $d_{D}^{+}(u)$. Hence, the total number of yet 
undominated neighbours of $u$ is at most $d_{H}(u)-\zeta(u)-d_{D}^{+}(u)$ which is the capacity of $u$. Hence $S$ is a capacitated dominating set of $H$.

Lemma 4. If $H$ has a capacitated dominating set $S$ of size $k^{*}$ then $D$ has a satisfying supply selection.

Proof. There are two kinds of unmarked vertices in $H$, list vertices and vertices of degree 2. Every degree 2 vertex $u$ has exactly one neighbour that is unmarked, and one neighbour $v$ that is a list vertex. Since the capacity of $v$ is equal to its degree and all marked vertices must be in $S$, if $u \in S$ then $S \cup\{v\} \backslash\{u\}$ is a capacitated dominating set of $H$ of size at most $k^{*}$. Thus, without loss of generality, all unmarked vertices in $S$ are list vertices.

For an arc $u v$ of $D$, let $s(u v)$ be the number of vertices in $S$ in the gadget corresponding to the arc $u v$. For a vertex $u$ of $D$ let $s^{+}(u)=\sum_{u v \in A(D)} s(u v), s^{-}(u)=$ $\sum_{v u \in A(D)} s(u v)$ and $s(u)=s^{+}(u)+s^{-}(u)$. Since $S$ contains at most $|A(D)|$ unmarked vertices we have that $\sum_{u \in V(D)} s(u) \leq 2|A(D)|$. If $s(u)<d_{D}(u)$ for a vertex $u$ then the number of vertices in $N_{H}(u)$ dominated by vertices other than $u$ is at most $s(u) \cdot(\mathcal{D}+$ $\left.n^{1} 0\right)<d_{D}(u) M$. However the capacity of $u$ is at most $d_{H}(u)-d_{D}(u) M$, contradicting that $S$ is a capacitated dominating set. Hence, for every node $u \in N(D), s(u) \geq d_{D}(u)$. If for some node $s(u)>d_{D}(u)$ then $\sum_{u \in N(D)} s(u)>\sum_{u \in N(D)} d_{D}(u)=2|A(D)|$, contradicting that $\sum_{u \in N(D)} s(u) \leq 2|A(D)|$. Thus, for every node $u \in N(D), s(u)=d_{D}(u)$.

Consider now three consecutive arcs $p q, q r$ and $r s$ in $A(D)$ such that both $q$ and $r$ have degree 2 in $D$. There are three cases, either $s(p q)=s(q r)=s(q s)=1$ or $s(p q)=s(q s)=2$ and $s(q r)=0$ or finally $s(p q)=s(q s)=0$ and $s(q r)=2$. We show that the last two cases lead to a contradiction. If $s(p q)=s(q s)=2$ and $s(q r)=0$ then the number of neighbours of $r$ dominated by vertices other than $r$ is at most $2\left(\mathcal{U}+n^{1} 0\right)<2 M$. However the capacity of $r$ is at most $d_{H}(r)-2 M$, contradicting that $S$ is a capacitated dominating set. Similarly, if $s(p q)=s(q s)=0$ and $s(q r)=2$ then the number of neighbours of $q$ dominated by vertices other than $q$ is at most $2\left(\mathcal{U}+n^{1} 0\right)<2 M$. However the capacity of $q$ is at most $d_{H}(q)-2 M$, contradicting that $S$ is a capacitated dominating set. It follows that $s(p q)=s(q r)=s(q s)=1$. Because the distance in $H$ between any pair of vertices with degree 4 is at least 3 it follows that $s(p q)=1$ for every arc $p q \in A(D)$.

We now make a supply selection $\left(f_{a}, f_{b}\right)$ for $D$ as follows. For every arc $u v$ there is exactly one unmarked vertex $x$ in $S$ in the gadget in $H$ corresponding to the arc $u v$. This vertex $x$ corresponds to a pair $(p, q) \in L(u v)$ and we make $u v$ select the pair $(p, q)$. Every arc selects a pair from its list in this manner. We now show that this supply selection is satisfying. Suppose for contradiction that this is not the case, then there is some vertex $u \in N(D)$ whose demand is not met. Then $u$ is a marked vertex in $H$, and the demand of $u$ is $d_{H}(u)-\zeta(u)-d_{D}^{+}(u)$. The number of neighbours of $u$ that are dominated by vertices other than $u$ is at most $\sum_{v \in N^{+}(u)} f_{a}(u v)+\sum_{v \in N^{-}(u)} f_{b}(v u)<\zeta(u)$. Since $s(p q)=1$ for every arc $p q \in A(D), u$ is adjacent to exactly $d^{+}(u)$ vertices in $S$. Thus $u$ must dominate more than $d_{H}(u)-\zeta(u)-d_{D}^{+}(u)$ vertices, a contradiction. This concludes the proof. 
The constructions together with Lemmata 1, 2, 3 and 4 yield the main result of this paper.

Theorem 1. Planar Capacitated Dominating Set is $W[1]$-hard.

\section{References}

1. J. Alber, H. L. Bodlaender, H. Fernau, T. Kloks and R. Niedermeier, Fixed Parameter Algorithms for DOMINATING SET and Related Problems on Planar Graphs. Algorithmica 33(4), 46-493, (2002).

2. J. Alber, M. R. Fellows and R. Niedermeier, Polynomial-time data reduction for dominating set. J. ACM, 51(3), 363-384, (2004).

3. E. D. Demaine, F. V. Fomin, M. T. Hajiaghayi and D. M. Thilikos, Subexponential parameterized algorithms on bounded-genus graphs and H-minor-free graphs. J. ACM 52(6), 866-893, (2005).

4. M. Dom, D. Lokshtanov, S. Saurabh and Y. Villanger, Capacitated Domination and Covering: A Parameterized Perspective. In the Proceedings of IWPEC, LNCS 5018, 78-90, (2008).

5. R.G. Downey and M.R. Fellows, Parameterized Complexity. Springer-Verlag, (1999).

6. Michael R. Fellows, Danny Hermelin, Frances A. Rosamond and Stéphane Vialette, On the parameterized complexity of multiple-interval graph problems, Theoretical Computer Science 410(1), 53-61, (2009).

7. J. Fiala, P. A. Golovach and J. Kratochvíl, Parameterized Complexity of Coloring Problems: Treewidth versus Vertex Cover. In the Proceedings of TAMC, LNCS 5532, 221-230, (2009).

8. J. Flum and M. Grohe, Parameterized Complexity Theory. Springer-Verlag, (2006).

9. F. V. Fomin, P. A. Golovach, D. Lokshtanov and S. Saurabh, Clique-width: on the price of generality. In the Proceedings of SODA, 825-834, (2009).

10. F. V. Fomin and D. M. Thilikos, Dominating Sets in Planar Graphs: Branch-Width and Exponential Speed-Up. SIAM Journal on Computing, 36(2), 281-309, (2006).

11. J. Guo, R. Niedermeier, Linear Problem Kernels for NP-Hard Problems on Planar Graphs. In the Proceedings of ICALP, LNCS 4596, 375-386 (2007).

12. R. Niedermeier, Invitation to Fixed-Parameter Algorithms. Oxford University Press, (2006). 